\title{
Proper Orthogonal Decomposition Based Turbulence Modeling
}

\author{
Mark N. Glauser \\ Assistant Professor \\ Dept. of Mechanical and Aeronautical Engineering \\ Clarkson University \\ Potsdam, NY 13699
}

August 6, 1991

\begin{abstract}
The proper orthogonal decomposition (v. Lumley 1967) is utilized to provide one mode estimates of various turbulence quantities in the near-wall region of the turbulent boundary layer. The experimental data of Herzog (1986) and the direct numerical simulation (DNS) results of Zang (1991) provide the two-point correlation tensor. The correlation tensor is then used as the kernel in the integral eigenvalue problem from which the proper orthogonal modes are obtained. The one mode estimates of the turbulent kinetic energy, dissipation, and triple moments all exhibit the proper asymtotic behavior near the wall. As a validation test, a model for the triple moments is developed and its near-wall behavior is studied. This information can then be used to obtain an estimate of the damping function near the wall, which is then compared to known existing behavior.

Since this is an initial investigation the streamwise and spanwise dependence is surpressed and only the wall normal direction $\left(y^{+}\right)$behavior of various turbulence statistics near the wall is studied. Before examining one mode estimates of the triple moments and the dissipation (on which the model is based), various second order moments are examined. If these do not exibit the proper behavior near the wall (as discussed by Speziale et at 1990) it does not seem reasonable to expect the one mode estimate of the triple moment and dissipation to have the right behavior. Only the results obtained using the experimental data of Herzog will be discussed here since the results from the simulations of Zang exhibit similar behavior.

In all of the figures discussed the dashed line corresponds to the one mode reconstruction of the particular quantity and the solid line to the proper behavior near the wall (v. Speziale et al 1990). Figure 1 shows the one mode estimate of the turbulent kinetic energy. Note how well the slope from the one mode estimate matches the proper behavior near the wall which for the kinetic energy is $y^{+2}$. Figure 2 shows the one mode estimate of the Reynolds stress compared to the proper behavior which is $y^{+3}$. Again as was observed with the energy, the one mode estimate of the Reynolds stress is doing a remarkable job.

Since the one mode estimates of the kinetic energy and Reynolds stress clearly exhibit the right behavior it seems reasonable to inspect the behavior of the one mode estimates
\end{abstract}


of the triple moment and the dissipation near the wall. Figure 3 shows the one mode estimate of the triple noment compared to the proper behavior which is $y^{+4}$. Here, as was observed with the second moments, the one mode estimale is doing quite well. Figure 4 shows a one mode estimate of the dissipation. It should be a constant near the wall. In the results shown here the dissipation is seen to increase slightly but this is well within the experimental accuracy of the data of Herzog. It should be noted that the DNS results of Zang for the dissipation were constant. Figure 5 shows the one mode estimate of the model near the wall. It, as well, exhibits the proper behavior which is $y^{+5}$.

Since the one mode estimates of the triple moment, the model and the dissipation all exhibit the proper behavior near the wall, the wall damping function can be obtained as well. The damping function is calculated by dividing the one mode estimate of the triple moment by that of the model. This result is shown plotted in figure 6 . Except for very near the wall the damping function has the right behavior which is $1 / y^{+}$. This deviation near the wall is due to experimental problems in this region and not deficiences in the approach. This was verified by utilizing the simulations where the one mode estimate of the damping function was seen to have the right belavior up to the wall.

Future work will involve extending these idcas to more complex turbulent flows such as separated shear layers and various compressilde flows where the proper behavior of the various quantities is not obvious. The ideas presented here then hold forth the possibility of helping us to predict more accurately these and other complicated turbulent flows.

\section{References}

Ilerzog, S. (1986), The large Seale Structure in the Near-wall Region of Turbulent Boundary Layer, Ph.D. thesis, (Cornell University.

Lumley, J.L. (1967), The Sitructure of Inhomogencous Turbulent Flows, Atmospheric Turbulence and Radio Wave Propagation, Moscow 1967.

Speziale, C.G., Abid, R., and Anderson, E.C., (1990), A Critical Evaluation of TwoEquation Models for Near Wall Turbulence, AIA $90-1481$.

Zang, T.A. (1991), private communication. 


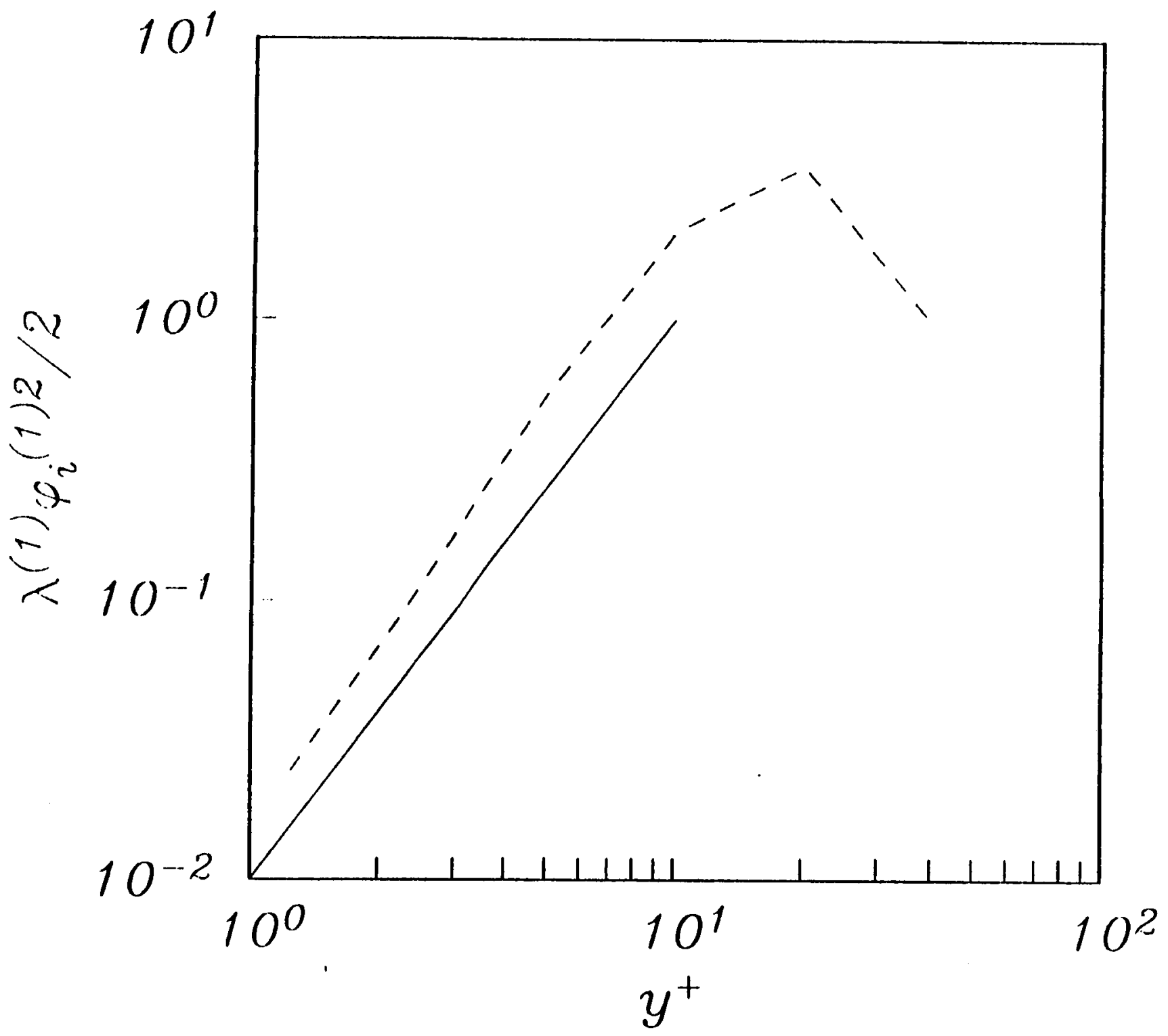

Figure 1. One mode contribution to the turbulent kinetic energy. 


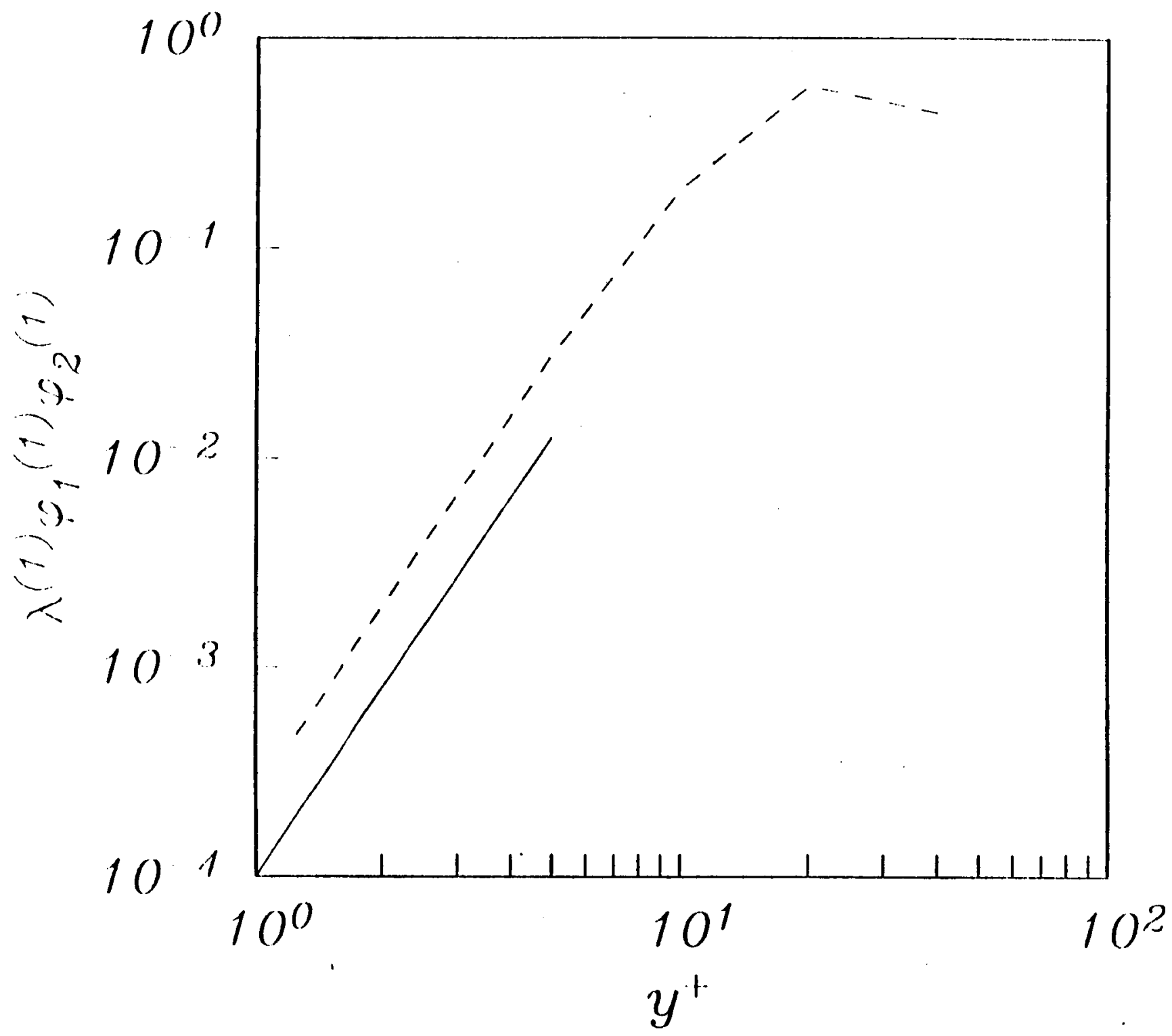

Figure 2. One mode contribution to the Reynolds stress. 


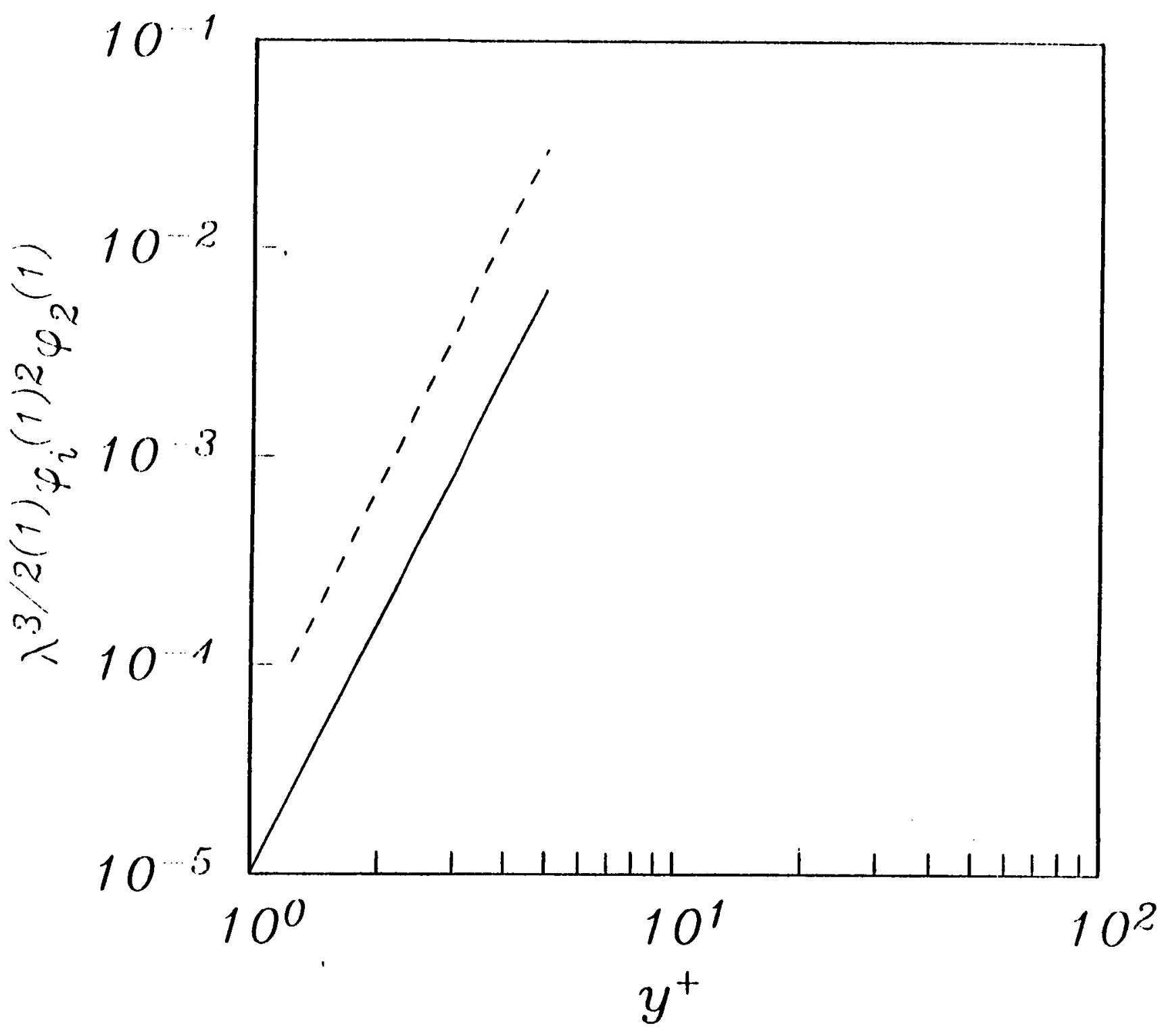

Figure 3. One mode contribution to the triple moment. 


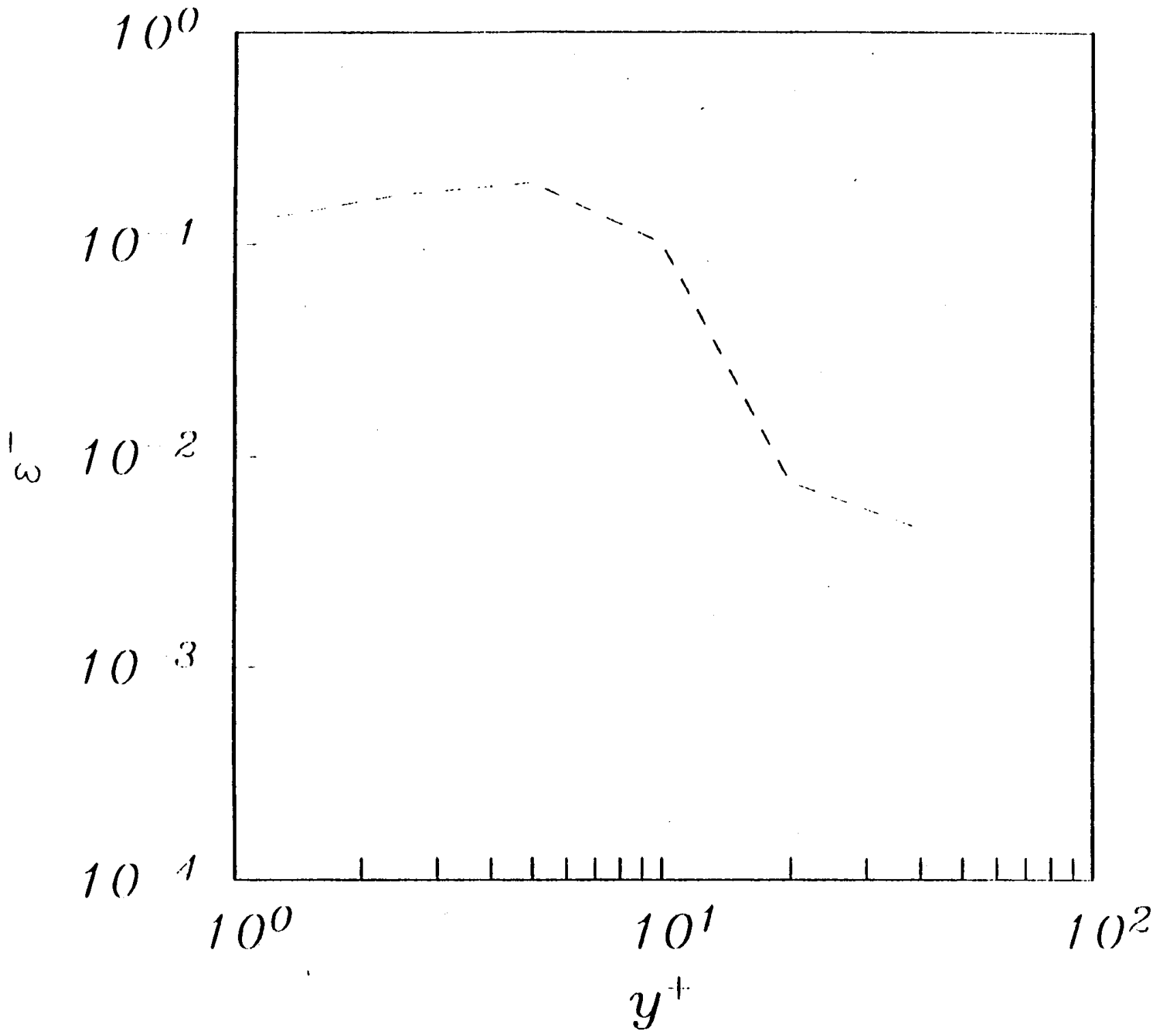

Figure 4. One mode contribution to the dissipation of kinetic energy. 


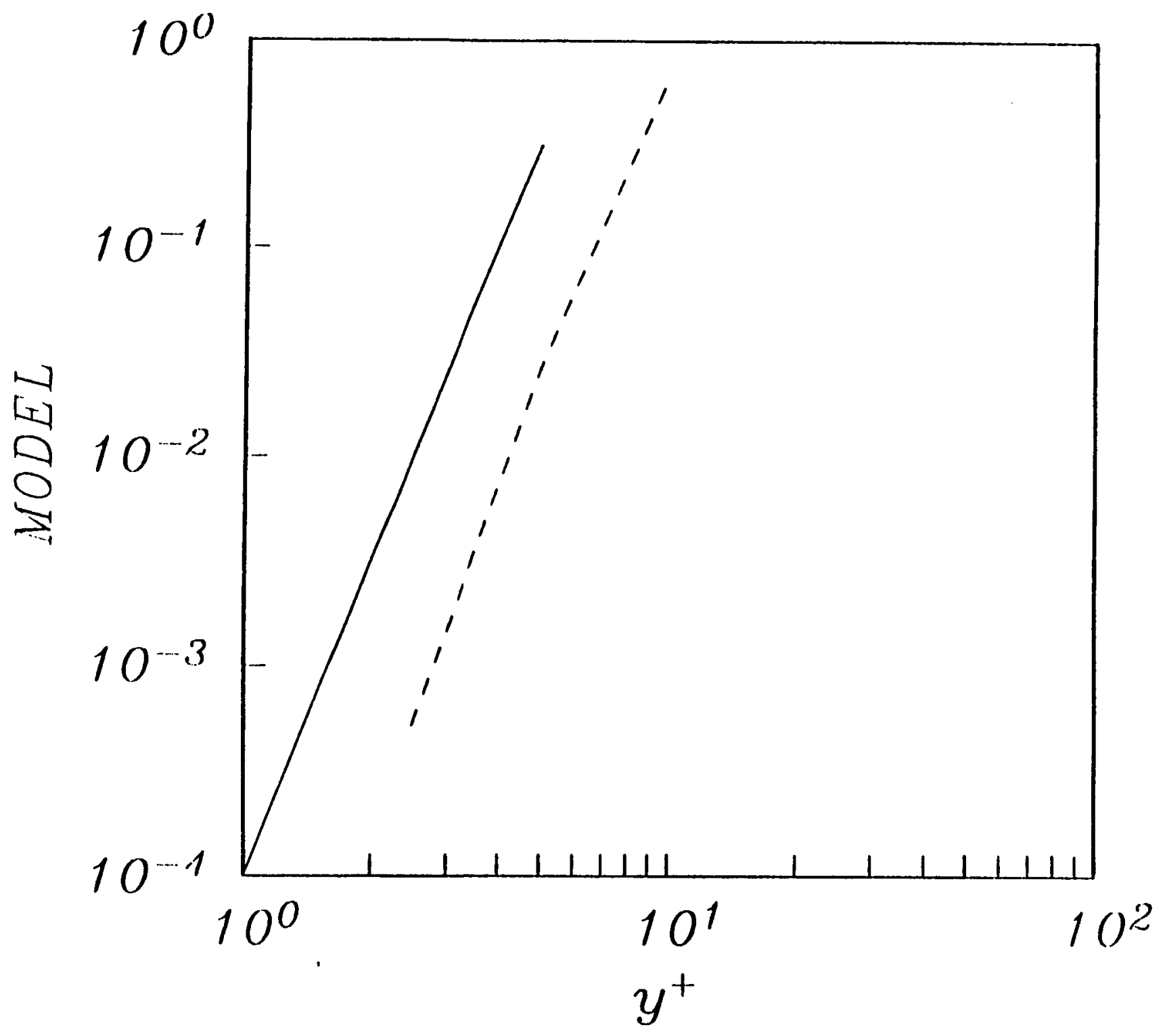

Figure 5. One mode contribution to the Model. 


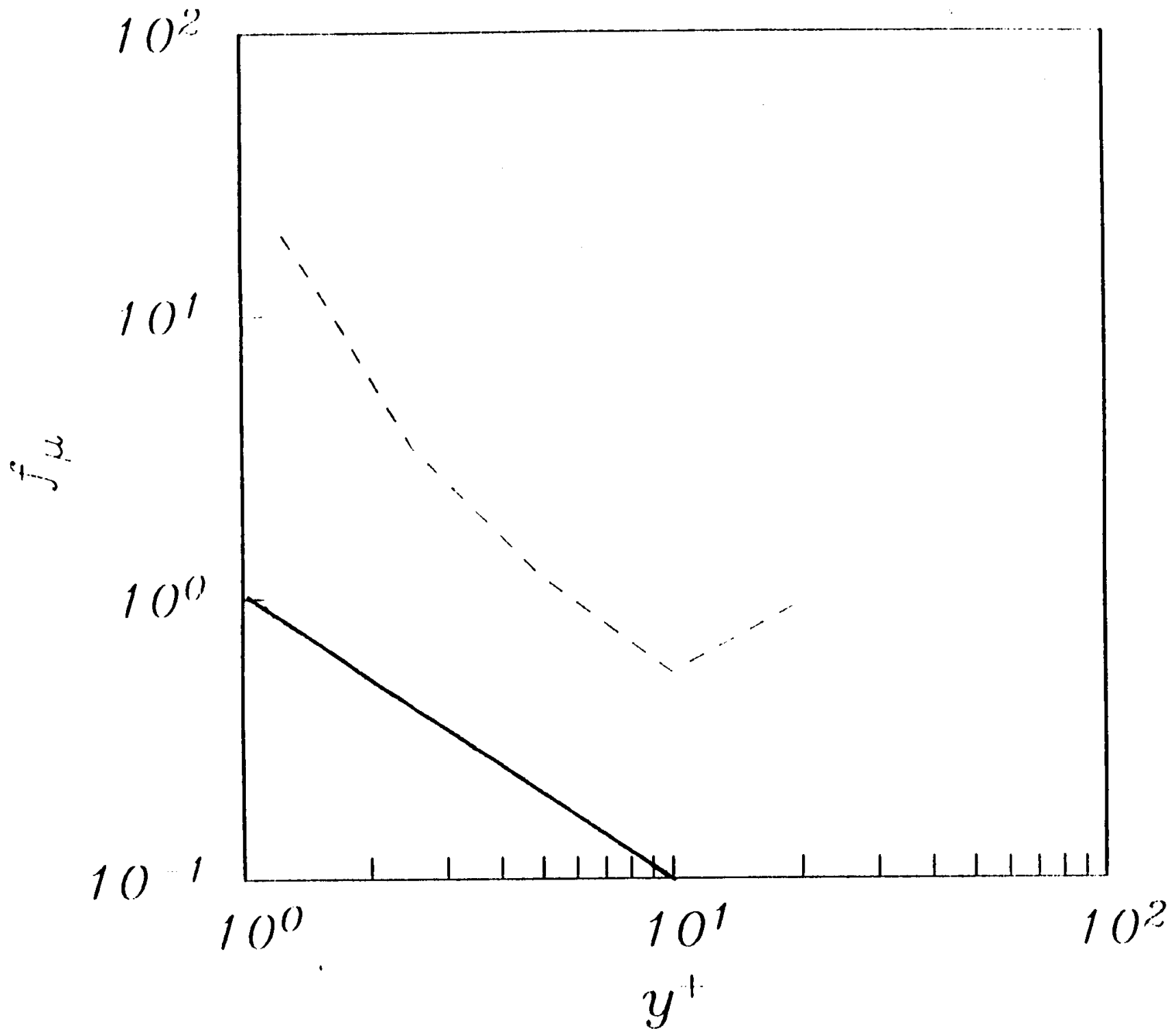

Figure 6. One mode contribution to the wall damping function. 